\title{
STORIA "INTERNA" E STORIA "ESTERNA" DEI TESTI: CoPERTINE DI ROMANZI IN ITALIA E IN BRASILE
}

\author{
Lucia Wataghin \\ SARA DebenedetTI ${ }^{*}$
}

ABSTRACT: L'articolo propone considerazioni sulla funzione delle copertine parte della storia "esterna" delle opere - di una serie di romanzi di autori dell'Einaudi, come Pavese, Ginzburg, Calvino e Levi, pubblicati anche in traduzione brasilana. Confrontare le copertine degli stessi romanzi nei due paesi stimola riflessioni sulla loro funzione come elementi delle strategie editoriali di vendite e come specchio del contesto sociale e culturale della ricezione delle opere.

PAROLE CHIAVE: paratesto; copertine; traduzione

ABSTRACT: O artigo propõe considerações sobre a função das capas - parte da história "externa" das obras - de uma série de romances de autores da Einaudi, como Pavese, Ginzburg, Calvino e Levi, publicados também em tradução brasileira. Comparar as capas dos mesmos romances nos dois países estimula reflexões sobre sua função como elementos das estrategias editoriais de venda e como espelho do contexto social e cultural da recepção das obras.

PALAVRAS-CHAVE: paratexto; capas; tradução

* Universidade de São Paulo, São Paulo (Brasil) - luciawataghin@gmail.com / sea.debenedetti@gmail.com Processo n.2013/20971-0 Fundação de Amparo à Pesquisa do Estado de São Paulo (FAPESP)

DOI: http://dx.doi.org/10.11606/issn.2238-8281.v0i33p69-83 
ABSTRACT: This article analyses the function of the covers - as part of the external story - of a series of Italian works, later translated into Portuguese and published in Brasil, written by the main authors at the Einaudi Publishing House in Italy, such as Pavese, Ginzburg, Calvino e Levi. This study compares and discusses the covers of these novels - in their original and translated versions - in both countries in order to reflect upon their role as editorial strategies to promote sales, but mainly as mirrors of their social and cultural context and their reception.

KEYWORDS: paratext; covers; translation 


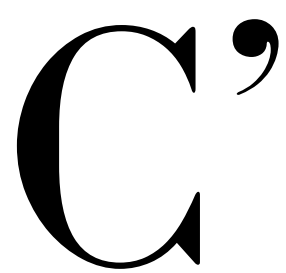

è una casa editrice italiana che ha raccolto, intorno alle sue pubblicazioni, un gruppo di intellettuali di tale rilievo, da raggiungere i più alti livelli di qualità editoriale, non soltanto nella scelta degli autori e delle opere da pubblicare, ma anche nelle scelte grafiche, ossia di presentazione fisica dei testi. Il risultato di queste scelte editoriali è il libro, quell'oggetto che, come diceva Calvino nel suo romanzo Se una notte d'inverno un viaggiatore, il lettore in un primo momento rigira fra le mani. E proprio Calvino fu uno degli intellettuali di quel gruppo, riuniti per lavorare alle pubblicazioni della casa editrice Einaudi, di Torino.

Testo e lettore si incontrano dunque nel libro. Tre sono le fasi di "convivenza" del lettore con il libro: in esse varia il rapporto tra l'elemento interno (il testo) e gli elementi esterni (il peritesto, secondo le definizioni di Gérard Genette), che si stagliano su piani differenti. Prima fase, la scelta: l'autore, il titolo, l'argomento sono fondamentali, ma non sempre sono da soli in primo piano, non sempre colpiscono separatamente dalla copertina - spesso arricchita da un'immagine - che li contiene e racchiude il testo. Capita che l'immagine di copertina raggiunga l'occhio del lettore in simultanea con il titolo e il nome dell'autore. Per Calvino è il momento del "girare intorno al libro, leggerci intorno prima di leggerci dentro". Seconda fase, la lettura: finiscono in ombra copertina, retrocopertina, risvolti, il testo balza in primo piano e calamita tutta l'attenzione del lettore. Terza fase, la conclusione della lettura: il testo si è concluso, o meglio, si è concluso il primo e fondamentale incontro del lettore con il testo e del testo con il suo lettore; il gesto concreto di chiusura del libro riporta l'occhio alla copertina, al titolo e all'immagine da 
cui era partito; ma ora questi elementi dicono altro, non anticipano bensì concludono il testo. Il libro si ricompone e, grazie alla sinergia di testo e peritesti, resta impresso nella mente del lettore. Quando questi vorrà cercare quel libro in mezzo a tanti, farà ricorso prima di tutto ai suoi elementi 'esterni': dimensioni, frontespizio e, soprattutto, immagine di copertina. ${ }^{1}$

Se un testo non esiste senza il supporto che lo offre alla lettura, ecco che il viaggio del testo verso i suoi lettori è anche il viaggio del libro, oggetto editoriale. È di una piccola parte di questo "viaggio" del libro nelle sue edizioni - italiane e brasiliane - che si tratterà nelle pagine che seguono, dando particolare attenzione ad alcuni elementi del peritesto, come la copertina e i risvolti.

Pensare al paratesto significa pensare alla storia esterna del testo, a ciò che succede quando il testo non è più nelle mani dell'autore, è stato consegnato all'editore e quindi al pubblico. Il paratesto stabilisce il primo patto con il ricettore, gli fornisce indicazioni di lettura, gli suggerisce interpretazioni. E fornisce - a livello di studio - dati sulle strategie messe in atto dall'editore per attrarre il lettore e, dopo un certo tempo, quando le strategie sono consolidate, un quadro di come un autore, e certi titoli, sono stati presentati e accolti in un dato sistema culturale. Confrontare le diverse copertine di diverse edizioni di un libro fa pensare a come cambiano gli stili degli editori e, parallelamente, i gusti del pubblico; in un senso ancor più ampio, al rapporto che la letteratura ha con la cultura in una data epoca o luogo.

È un affascinante percorso perché, se la creazione del testo è un atto unico, la produzione del libro, come suo supporto, è un atto che può ripetersi e variare nel tempo e nello spazio. Affermano Cavallo e Chartier nell'introduzione a Storia della lettura (2009):

Gli autori non scrivono libri: essi scrivono testi che diventano oggetti scritti manoscritti, incisi, stampati e, oggi, informatizzati - maneggiati in maniere diverse da lettori in carne ed ossa le cui modalità di lettura variano secondo i tempi, i luoghi, i contesti. ${ }^{2}$

1 Il critico Marco Filoni, nella rubrica Scopertine, pubblicata sul Venerdi di Repubblica, 26/02/2016, scrive: "Ci sono copertine che regalano un sapore. Palpabile proprio come il contenuto del libro. Diceva l'intellettuale ungherese Béla Balázs che i libri hanno il loro stato d'animo, la loro aura visibile proprio nella loro copertina, che forma un volto che riscalda il cuore".

2 Cavallo, Guglielmo, Chartier, Roger (a cura di). Storia della lettura. Bari: Editori Laterza, 2009. 
Non solo le modalità di lettura, anche le modalità di edizione variano secondo i tempi, i luoghi e i contesti. Esse sono strettamente legate una all'altra. L'importanza della veste con cui si presenta un libro, e che qui verrà presa in esame, non deve essere sottovalutata: il libro si presenta fisicamente, nel suo insieme, e il suo aspetto fisico riflette il giudizio, la valutazione che gli è stata data, prima di arrivare al pubblico, dal mondo di lettori specializzati che è il mondo editoriale. La veste del libro (la qualità della carta, la copertina, la cura dell'edizione) trasmette un'informazione anche sull'identità dell'editore, come sostiene Cadioli in Le diverse pagine (2012).

L'editore italiano di cui ci si occuperà in questo breve scritto è Einaudi perché, come anticipato, riunì intorno a sé, come redattori e collaboratori, grandi nomi della letteratura italiana del Novecento. La casa editrice Einaudi è stata a lungo prestigiosa, ha definito tendenze culturali, ha scelto, proposto e imposto titoli e autori in Italia. Per quanto riguarda la veste del libro, ha sempre presentato uno stile sobrio ma raffinato, che l'ha contraddistinta in tutte le collane e nel corso della sua storia di più di mezzo secolo. ${ }^{3}$

Qui si è deciso di prendere in esame un periodo particolare dell'Einaudi, il periodo del dopoguerra, e quattro autori, tre dei quali - Cesare Pavese, Natalia Ginzburg e Italo Calvino sono stati anche importanti collaboratori della casa editrice. Il quarto, Primo Levi, è stato molto vicino all'Einaudi, presso cui ha pubblicato quasi tutti i suoi libri (con un paio di eccezioni, tra cui quella del primo libro, Se questo è un иomo, che, come è noto, fu pubblicato per la prima volta da Franco Antonicelli nel 1947), comprese le traduzioni (per le Edizioni Scientifiche Einaudi). Scegliendo questi quattro autori si vuole solo proporre l'osservazione di campioni all'interno di un universo relativamente omogeneo (non certo considerare sistematicamente la storia di tutte le copertine e le quarte di copertina e i risvolti di tutti e quattro). Volendo inoltre seguire una parte del percorso che questi libri hanno fatto non solo nel paese di origine, ma anche in Brasile, la scelta dei quattro autori si giustifica per il numero consistente di edizioni che hanno avuto in questo paese. Insieme alle edizioni italiane, permettono uno studio più ampio del rapporto fra storia "interna" e storia "esterna" del testo.

La terza edizione italiana di Paesi tuoi, di Cesare Pavese, è del 1945. Nella copertina, estremamente sobria in assenza di illustrazioni, spiccano il titolo e lo struzzo einaudiano. I

3 In occasione del primo cinquantenario della casa editrice, è stato pubblicato il libro Cinquant'anni di un editore. Le edizioni Einaudi negli anni 1933-1983. In esso la prima sezione è costituita da una breve storia attraverso l'iconografia, che include molte copertine delle varie collane pubblicate nel corso di quegli anni. 
Dialoghi con Leucò esce nella collana dei saggi: una cornice arancione inquadra nome dell'autore, titolo, editore e marchio su sfondo bianco. L'ultima edizione del 2016 ha un particolare da Le tre grazie, di Antonio Canova. Una pari sobrietà - e al tempo stesso eleganza - caratterizza l'edizione brasiliana di Diálogos com Leucó (2001), pubblicata dalla Cosac \& Naify: sulla sovracoperta l'immagine del Tuffatore di Paestum. Le immagini di entrambe le edizioni, italiana e brasiliana, hanno un riferimento al contenuto del testo. È interessante notare che, nell'edizione della Cosac \& Naify, sulla copertina grigia spiccano solo le iniziali CP, in basso il nome della casa editrice. Ma aprendo il libro, ecco nella pagina a sinistra del frontespizio una fotografia dell'autore, e nella pagina a sinistra della prefazione (a cura di Maurício Santana Dias) una foto del paese di S. Stefano Belbo.

Le successive collane di narrativa dell'Einaudi corredano copertine o sovracoperte con riproduzioni di opere di grandi artisti, scelta che identifica sia l'editore sia, in certi casi e in certi accostamenti, perfino lo scrittore. Proprio Pavese viene spesso abbinato a uno stesso pittore, Van Gogh. Dialoghi con Leucò, nell'edizione dei Nuovi Coralli del 1979 ha un particolare del quadro Campo di grano con corvi; La casa in collina ha i Campi di grano; Il compagno ha Il ponte della ferrovia sull'avenue Montmajor (in un'edizione degli Einaudi Tascabili del 2007 il quadro è Il caffé di notte); Il carcere ha Le cisterne del gas (in un'altra collana è invece illustrato con il dipinto di Edita Broglio Pane e acqua (1926): un bicchier d'acqua e un cornetto di pane appoggiati su una tovaglia bianca).

A proposito della scelta delle immagini per le copertine, lo stesso Pavese scriveva (Antologia Einaudi, 1948):

alla prima veste dei "Coralli" affidata a Cassinari, Morlotti, Guttuso, Ajmone, ecc. successe l'attuale: la nuova rilegatura, l'attenta ricerca di un particolare, di una decorazione da un grande quadro di Renoir, di Daumier, di Picasso, di Van Gogh, di Carrà, di Matisse, di Chagall, di Moore, di quanto di meglio offre la pittura dell'ultimo secolo. È un sottile lavoro d'interpretazione e illuminazione: sovente un accostamento tra uno scrittore e un gusto figurativo preesistente vale un intero saggio critico. ${ }^{4}$

4 Pavese, Cesare. Antologia Einaudi 1948. Apud Cadioli, Alberto. Le diverse pagine. Milano: Il Saggiatore, 2012, pag. 203. 
Di questo lavoro editoriale di scelta delle copertine einaudiane ci sono parecchie testimonianze, tra cui quelle di Ernesto Ferrero, che per molti anni ha lavorato nell'Einaudi, di cui è stato anche direttore:

Forse non tutti sanno che l'incantamento di tante copertine e sovraccoperte Einaudi era opera di Giulio Bollati. [...] Ebbene, in questi cortocircuiti letterario-figurativi il Maestro era insuperabile. Si chiudeva nella stanzina-biblioteca accanto allo studio dell'editore, tirava giù dagli scaffali dei grossi tomi illustrati, si aiutava con dei fogli di carta a isolare quel certo particolare significante, il taglio che dava all'immagine una suggestione nuova e imprevista, ed ecco trovata in dieci minuti la copertina «giusta». Solo chi ha avuto la fortuna di vederlo lavorare in quel modo può sapere quanta intelligenza critica, quanta sicurezza di gusto stavano nella naturalezza con cui eseguiva le sue invenzioni grafiche. ${ }^{5}$

Ma da dove nasceva questa particolare attenzione per le copertine? Nella sua breve storia dell'Einaudi, I migliori anni della nostra vita (2005), Ferrero racconta:

Era stato Attilio Bertolucci, professore di liceo di Bollati, a Parma, a suggerirgli di utilizzare la grande pittura per offrire al lettore una chiave interpretativa preliminare. Bollati sceglieva opere che esprimessero una comune sensibilità, un'affinità di spirito: Van Gogh per Pavese, Klee per Calvino, Picasso per Hemingway. [...] In dieci minuti aveva trovato la copertina capace di suggerire la qualità del libro che stavi per leggere. ${ }^{6}$

In realtà si sa che Calvino sceglieva da sé tutte le copertine dei suoi libri e il pittore che prevale nelle sue scelte - come si vedrà in seguito - è Picasso (Barenghi, curatore del Meridiano di Calvino, dice che Picasso nelle copertine di Calvino ha valore paradigmatico ${ }^{7}$ ).

Tornando a Pavese, in Brasile l'Editora Brasiliense pubblica O belo verão (traduzione de $\mathrm{La}$ bella estate) nel 1987 e Mulheres sós (traduzione di Tra donne sole) nel 1988. La casa editrice,

5 Ferrero, Ernesto, http://www.einaudi.it/speciali/Einaudiani.-Ernesto-Ferrero-su-Giulio-Bollati 6 Ferrero, Ernesto. I migliori anni della nostra vita. Milano: Feltrinelli, 2005, pag. 46.

7 Barenghi, Mario. Italo Calvino, le linee e i margini, 2007. Apud Cadioli, Alberto, Le diverse pagine, cit., pag. 203. 
fondata da Caio Prado e diretta a lungo da Monteiro Lobato, è una delle più tradizionali. Ha pubblicato - oltre a Pavese - Pasolini, Goldoni, Dacia Maraini, Trilussa. Le copertine dei libri di Pavese sono opera dell'artista grafico Roberto Emílio Nejme: pochi tratti e colori che illustrano, quasi in modo onirico, una collina, tre alberi e tre figure umane con le rispettive ombre, proiettate da un sole ovale sospeso nell'azzurro; i busti di due donne, una quasi ombra dell'altra. Si è cercato, in queste edizioni, un rapporto diretto non solo con la storia interna, ma prima di tutto con il titolo delle rispettive opere.

L'edizione brasiliana del 2009 di Trabalhar cansa (Lavorare stanca) è di nuovo della Cosac \& Naify; ha una copertina dove risaltano - oltre al nome completo dell'autore e al titolo - la trama del cartoncino (la materia prima della copertina) e la trama di una pagina autografa delle poesie (la materia prima del testo); i rifacimenti e le molteplici cancellature (la pagina sembra del tutto annullata) non possono non far pensare alla faticosa opera di elaborazione che sta dietro alla poesia. La casa editrice sembra confermare, anche con questo titolo, che punta sugli intenditori.

Natalia Ginzburg entrò nel "cantiere" Einaudi subito dopo la guerra, lavorando a fianco di Pavese, che aveva fatto parte del gruppo dei fondatori ed era alla guida della redazione torinese. Come scrittrice, pubblicò sempre i suoi libri da Einaudi e la stessa casa editrice li ripubblicò in anni successivi e in altre collane. Ciò ci permette di fare alcune osservazioni e supposizioni sulle scelte editoriali.

Innanzi tutto, osservando le copertine, si nota che non c'è un accostamento a uno specifico pittore, come in Pavese, segnale di un suggerimento di affinità tra scrittore e pittore. C'è piuttosto un segnale che rimanda alle storie interne dei testi, ai loro protagonisti, alle vicende che vi si narrano. E, spesso quando pubblicati in altre collane, un segnale che rimanda al tipo di lettore a cui è diretto il libro. ${ }^{8}$ Per illustrare le copertine dei libri di Ginzburg, Einaudi sceglie diversi pittori, soprattutto francesi e italiani, dell'Ottocento e Novecento: Il ballo di Renoir per la commedia Ti ho sposato per allegria; gli ambienti interni, familiari di Bonnard, Matisse, Léger per Valentino, Romanzi brevi, Le voci della sera; è una scena d'interno anche quella sulla copertina di Caro Michele, di Jessie Boswell: in essa spicca una figura femminile che legge seduta in poltrona (sarà un caso che la pittrice sia nata a Leeds, in Inghilterra, dove si rifugia il protagonista Michele quando lascia Roma?); paesaggi urbani come quello di Bonnard

8 Einaudi ha una collana specifica per la scuola, in cui pubblica i grandi classici della letteratura adatti ai ragazzi delle scuole secondarie di I e II grado. 
(Maison dans la court) per Mai devi domandarmi e quello del pittore torinese Francesco Garrone (Antiche case di Torino) per un'edizione di Lessico famigliare nella collana Einaudi Scuola; ritratti di Modigliani e di Paulucci, rispettivamente per due edizioni di $\grave{E}$ stato così e una seconda di Valentino, e per La strada che va in città; il particolare da un'opera di Egon Schiele, quello di una ragazza semisdraiata vista di schiena, illustra la copertina di Lessico famigliare in tutte le molteplici edizioni (salvo quella scolastica, come si è visto). Questi sono esempi di "grande pittura per offrire al lettore una chiave interpretativa preliminare", di come si possano ricercare personaggi, ambienti e situazioni del testo interno in quello esterno. È il sottile lavoro di interpretazione di cui parlava Pavese. L'editore conta su un pubblico che conosca, riconosca e apprezzi l'opera (o il particolare) riprodotta in copertina, con la quale il lettore apre e chiude il circolo di lettura.

In Brasile sono stati pubblicati ben undici libri di Natalia Ginzburg, il primo, Le voci della sera (1952), tradotto con il titolo A vida não se importa nel 1960, l'ultimo, Le piccole virtù (1962), As Pequenas Virtudes, nel 2015. Dieci anni separano la pubblicazione di queste due opere in Italia, cinquantacinque in Brasile. Dopo la prima edizione de Le piccole virtù, pubblicata nella collana dei saggi con l'austera copertina dalla cornice arancione e senza immagini, tutte le altre edizioni italiane hanno sulla copertina una foto dell'autrice, caratteristica solo di questo libro; e non stupisce, perché l'autrice è, più che mai, dentro questo libro. Sulla copertina dell'edizione brasiliana, invece, una mappa delle strade di Borgo Po e Oltrepò a Torino (dove inizia la collina), cui si sovrappone, sfumata, una foto in bianco e nero di un salotto torinese degli anni '50 - '60. Il richiamo è forse a uno dei saggi del libro, Ritratto di un amico, dedicato a Cesare Pavese, che così inizia: "La città che era cara al nostro amico è sempre la stessa", continua: "La nostra città rassomiglia, noi adesso ce ne accorgiamo, all'amico che abbiamo perduto e che l'aveva

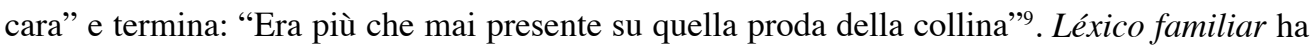
avuto due edizioni. La prima, dell'editore Paz e Terra (1988), ha sulla copertina una bambina che tira fuori dal vestito foto, oggetti di casa e lettere dell'alfabeto: c'è un richiamo ai temi, ma lo stile sembra lontano dal carattere del testo. La seconda (2009), dell'editore Cosac \& Naify, invece, è di tutt'altro stile: copertina rigida, uno scozzese monocromatico marrone, a piccoli caratteri il titolo e a grandi caratteri bianchi il nome N. Ginzburg. Interessante notare che sul dorso il nome che appare è Natalia G. Copertina e dorso si complementano in questo caso, in

9 Ginzburg, Natalia. Le piccole virtù. Torino: Einaudi, 1962, pagg. 25, 26, 32. 
un gioco interessante di rimandi. Il libro fa parte della Coleção Mulheres Modernistas: un autore è riconosciuto per il suo cognome, ma che si tratti di una scrittrice ce lo dice il nome. ${ }^{10}$ Osservando le due edizioni, possiamo supporre che non si tratti esclusivamente di differenze editoriali dovute all'identità dell'editore, ma anche ai nuovi lettori che si sono formati nei vent'anni che separano le due edizioni.

Italo Calvino, come consulente e poi direttore dell'Einaudi, è stato anche lui vicino alle scelte editoriali delle copertine, come si è visto. Ne parla, per esempio, nella presentazione alle Fiabe, di Grimm, illustrate già in copertina, da un particolare di Bruegel:

Le tavole a colori, tratte da quadri del pittore fiammingo cinquecentesco Pieter Bruegel il Vecchio, valsero a sottolineare attraverso una libera associazione d'immagini il ritmo che governa il mondo realistico-grottesco della fantasia contadina nordica. ${ }^{11}$

$\mathrm{Ci}$ aveva pensato, nella sua prima edizione, Giulio Bollati insieme a Elio Vittorini. In una lettera di questi indirizzata a Giulio Einaudi si legge:

Caro Giulio, pensando a Rabelais credo che il pittore più adatto a illustrarlo sia proprio Brueghel, [...] Si dovrebbe trovare un modo speciale di tagliare $\mathrm{i}$ particolari, perché il Brueghel col quale illustreremo Rabelais risultasse tutt'altro pittore da quello col quale abbiamo illustrato Grimm. ${ }^{12}$

È Bollati che, nel suo ritratto dell' "einaudiano" Calvino, afferma: "In più Calvino aveva una disinvolta familiarità e un occhio sicuro per le immagini, ed era all'occorrenza un ottimo copertinista [...] Mi piacerebbe cercare tutte le copertine fatte da lui o insieme a lui." 13

A proposito di fiabe, è interessante notare che le varie edizioni Einaudi delle Fiabe italiane, raccolte e trascritte da Calvino, hanno in copertina delle miniature di vita campestre o di corte (XII-XIV sec.), riprese dal grafico brasiliano Hélio de Almeida sulla laterale sinistra della

10 Nella Coleção Mulheres Modernistas è stato pubblicato anche Caro Michele (2010), sempre della Ginzburg.

11 Grimm, J. e W. Fiabe. Torino: Einaudi, 1970, pag. XVIII.

12 Vittorini, Elio. Gli anni del "Politecnico". Lettere 1945-1951. Torino: Einaudi, 1977.

13 Bollati, Giulio. http://www.einaudi.it/speciali/Einaudiani-Giulio-Bollati-su-Italo-Calvino. 
copertina delle edizioni brasiliane Companhia das Letras.

Si osservino ora alcune copertine dei più noti libri di Calvino, quelle che permetteranno anche un confronto con le edizioni brasiliane. Le copertine o sovracoperte di Il barone rampante, Il visconte dimezzato e Il cavaliere inesistente, che nell'edizione del 1962 sono raccolti col titolo I nostri antenati, sono illustrate con riproduzioni di disegni di Pablo Picasso. ${ }^{14}$ Cadioli, dopo aver riportato l'osservazione di Mario Barenghi sulle copertine di Calvino, secondo la quale esse erano "frutto di una decisione che era sempre anche sua", riflette sull'interpretazione che l'autore offre dei propri testi attraverso questa scelta editoriale. Difatti le differenti immagini di copertina che hanno caratterizzato le opere di Calvino in anni successivi (immagini più "piene" prima, più rarefatte dopo), mantengono tuttavia elementi costanti: "la volontà di intersecare piani di realtà diversi e diversi paradigmi formali, il gioco tra realtà e finzione, la riflessione sui linguaggi", ${ }^{15}$ perché sono elementi che caratterizzano i testi di Calvino, chiavi interpretative delle singole narrazioni.

Anche nelle edizioni brasiliane della Companhia das Letras notiamo differenti fasi di copertine. La prima, degli anni novanta, più figurativa, riprende elementi espliciti dei testi, come il cavaliere, l'albero, figure preistoriche e mitologiche, carte di tarocchi, ecc. Ne è autore Hélio de Almeida. Siamo negli anni in cui gli autori italiani - e forse fra i contemporanei prima di tutto proprio Calvino - si fanno conoscere al grande pubblico e si aprono un mercato. La varietà dei disegni insieme all'omogeneità dello stile delle copertine ne fanno delle edizioni sobrie ed eleganti allo stesso tempo, adatte a presentare questo autore. La seconda fase, degli anni duemila, si distingue per le copertine monocromatiche opache, con parallelepipedi o altre figure geometriche di misure differenti, lucide, disegnate da Raul Loureiro; quello che colpisce sono i titoli, presentati come titoli di una bibliografia: Cognome, nome dell'autore. Titolo. Casa editrice, anno. Titolo originale e anno di pubblicazione dell'opera. Un richiamo agli studi universitari sull'autore, sempre più frequenti in questi anni? E quindi edizioni che prevedono un pubblico più specialistico? A un pubblico più ampio si rivolgono le edizioni economiche Companhia De Bolso, della stessa casa editrice - le cui copertine sono disegnate da Jeff Fisher. Esse presentano a caratteri giganti il nome di Italo Calvino, seguito dal titolo e, in certi casi, da uno schizzo. Esempio interessante di un legame fra edizioni italiane e brasiliane è quello di $O$

14 http://www.internetculturale.it/opencms/opencms/it/pagine/mostre/pagina $190 . \mathrm{html}$

15 Barenghi, Mario, apud Cadioli, Alberto. Le diverse pagine, cit., p. 205. 
cavaleiro inesistente, che raffigura l'elmo vuoto, simile a quello del disegno originale creato da Picasso per la copertina de Il visconte dimezzato; del disegno di Picasso è stato ripreso anche il colore turchese di sfondo.

I libri di Primo Levi in Italia continuano ad essere pubblicati da Einaudi. In Brasile gli ultimi titoli sono stati pubblicati dalla Companhia das Letras, grande casa editrice che, nei suoi trent'anni di vita, ha cercato di ampliare il suo pubblico mantenendo una qualità editoriale e una scelta di titoli di prestigio: Se não agora, quando? (1999), A trégua (1997 e 2010), 71 contos de Primo Levi (2005), A chave estrela (2009), Assim foi Auschwitz (2015).

Tutte le copertine sono a cura di Jeff Fisher o di Victor Burton; quest'ultimo iniziò la sua carriera presso l'editore italiano Franco Maria Ricci, che pubblica edizioni d'arte e letterarie di pregio. Burton si serve di riproduzioni di quadri, come quella di Fernand Léger per A chave estrela. È, tra l'altro, una copertina molto simile a quella dell'Einaudi per La chiave a stella nell'edizione del 1979. Nella copertina di A trégua del 1997 si vede sullo sfondo di un cielo pallido il tratto bianco di un disegno di Chagall: l'uomo che vola sopra la casa. Qui la relazione che l'editore ha voluto suggerire fra storia interna e storia esterna appare evidente. Tra le edizioni degli anni Novanta e quelle degli anni più recenti notiamo che in primo piano appare il nome dell'autore e in secondo il titolo del testo: è possibile supporre che questo spostamento di piani sia il riflesso della fama acquisita da Primo Levi sia a livello internazionale sia presso il pubblico brasiliano. L'immagine di sfondo che occupa l'intera copertina di tre editori differenti - Rocco, Paz e Terra, Companhia das Letras - e che ha una diretta relazione con il tema principale del testo (per esempio la giacca a righe e il numero dei deportati, la piantina del campo di Auschwitz) ha forse lo scopo di richiamare l'attenzione di un pubblico che, per una questione di generazione, è sempre più vicino alla cultura dell' immagine e nello stesso tempo più lontano dagli avvenimenti narrati. ${ }^{16}$

Interessante è anche l'edizione brasiliana de L'ultimo Natale di guerra, pubblicata da Berlendis \& Vertecchia con il titolo O último Natal de guerra (2002), non tanto per la copertina di Rubens Ianelli, quanto per un altro paratesto, il risvolto di prima di copertina. Libro postumo,

16 Dobbiamo però rilevare, da parte della casa editrice brasiliana Companhia das Letras, una certa superficialità nell'edizione del 2015 di Assim foi Auschwitz. Testemunhos 1945-1986, che Primo Levi scrisse in parte insieme al compagno di prigionia Leonardo De Benedetti: proprio sulla copertina il cognome del medico appare come "Benedetti". Si tratta in questo caso di una doppia perdita d'identità, la prima anagrafica, la seconda religiosa, poiché De Benedetti è un cognome ebreo, Benedetti no. Potremmo dire che non si guarda mai abbastanza la copertina dei libri! 
curato nell'edizione italiana da Marco Belpoliti, mantiene nel risvolto dell'edizione brasiliana una parte interessante del testo di Belpoliti che definisce Levi "um híbrido, ou melhor, um centauro, como ele amava se definir". E in quarta di copertina un brano di uno dei racconti, richiamo del lettore per mezzo della scelta di un testo in ambito nettamente fantascientifico.

Levi è un esempio di autore italiano che continua a suscitare l'interesse di varie case editrici brasiliane, ultima in ordine di tempo la Unesp, che nel 2016 ha pubblicato A assimetria e a vida. Artigos e ensaios 1955-1987 e $O$ ofício alheio.

L'associazione proposta dalla Einaudi fra i suoi autori e opere pittoriche di alta qualità (e, più recentemente, con la moderna arte della fotografia giornalistica o cinematografica) è uno degli elementi messi in campo per consolidarne l'immagine come appartenenti all'alta letteratura. Per restare nell'ambito della storia "esterna" del libro, la buona qualità della carta, dell'impaginazione e in generale del lavoro grafico, oltre alla aperta segnalazione di qualità costituita dall'inserzione di ciascuno nell'opportuna collana, presentano queste opere e questi autori in maniera allo stesso tempo esplicita e subliminale. Esplicita perché si propone apertamente alla vista di tutti, ma subliminale perché l'impressione che si fissa nella memoria del pubblico non è direttamente legata al testo, ma è piuttosto quella di una serie di immagini visive esterne al testo. Gli autori da noi presi in considerazione sono strettamente legati, agli occhi del pubblico italiano, naturalmente con alterne vicende, alla "marca" Einaudi (quasi esclusivamente, con poche eccezioni), che conferisce loro il proprio prestigio, ricevendone in cambio altro prestigio.

In Brasile, Pavese, Ginzburg, Calvino e Levi hanno avuto e hanno tuttora fortune diverse. La grandissima fortuna di Calvino in Brasile ha prodotto un altrettanto grande varietà di edizioni e quindi anche di copertine, che hanno in comune una sobrietà ed eleganza sicuramente collegate alle corrispondenti edizioni einaudiane e hanno contribuito a consolidare e divulgare l'immagine sobria ed elegante dello scrittore italiano. La fortuna dei romanzi di Pavese, legata alla casa editrice Brasiliense negli anni '80, non è sostenuta e subisce un crollo da cui è parzialmente riscattata dall'editore Berlendis, che pubblica La Luna e i falò nel 2002. Ma un altro Pavese, l'autore più difficile e ricercato delle poesie di Lavorare stanca (2009) e dei Dialoghi con Leucò (2011), è riproposto nelle splendide edizioni Cosacnaify, che sottolineano appunto l'aspetto più grave e sostenuto dello scrittore. Resta ricercatissimo nel mercato dell'usato il volume dei diari, Il mestiere di vivere, nella traduzione di Homero Freitas de Andrade (ed. Bertrand Brasil, 1988), segnalando il perdurante interesse (non soddisfatto) del pubblico per il lato personale delle 
vicende e per la forma del diario. Osserviamo che in tempi diversi (tutta la fortuna di Pavese è concentrata negli anni ' 80 e ripresa solo con il recente rilancio della Cosacnaify) le edizioni hanno privilegiato aspetti diversi dello stesso autore e che ad ogni aspetto è corrisposta una particolare forma grafica e uno specifico paratesto (è parso opportuno, forse necessario, corredare con ampie posfazioni e risvolti di copertina firmati i volumi del Pavese poeta e autore dei sofisticati Dialoghi di Leucò, mentre per presentare i romanzi sono sufficienti copertine vivaci).

Quella parte della fortuna di Natalia Ginzburg costruita in Italia grazie alla complessa strategia visuale sopra descritta (suggestioni di austerità e alta cultura, abbinamenti dei suoi testi con quadri d'autore, insistenza sulle caratteristiche personali dell'autrice, in quanto donna, ma anche sugli aspetti semplicemente biografici della sua opera) è ripresa in parte anche in Brasile. Appare particolarmente sottolineato, in certe copertine, ciò che è personale e biografico, e quindi, quasi direttamente, l'appartenenza dell'opera all'universo femminile; questi forse sono motivi forti che hanno contribuito al fatto che Natalia Ginzburg sia conosciuta e amata, in misura crescente, in Brasile, come dimostra il grande numero di traduzioni.

Primo Levi, che potremmo definire un "autore Einaudi" in quanto pubblicato in Italia quasi esclusivamente da questa casa editrice, appartiene però, nella memoria collettiva brasiliana, più che alla Einaudi alla grande famiglia della letteratura di testimonianza e i suoi grandi libri sulla Shoah (Se questo è un uomo, La tregua, Se non ora, quando?) presentano copertine desolanti, legate a immagini di guerre, filo spinato, prigionieri, climi freddissimi. In entrambi i paesi si nota però in tempi recenti che si è passati dal gusto per l'immagine pittorica a una preferenza, diremmo epocale, per l'immagine fotografica, che appare ora il miglior modo di rappresentare, o alludere alla Shoah.

Nel percorso studiato delle copertine dei testi di Pavese, Ginzburg, Calvino e Levi è emerso il rapporto che esse hanno con il paese di edizione, ossia con la cultura e il gusto sia dell'editore che del suo pubblico e lo stretto rapporto che le lega a un determinato momento socio-culturale. Oggi le edizioni Einaudi - filo conduttore di questo articolo - accanto ai grandi della pittura, collocano i grandi della fotografia d'autore e, quando dal libro è stato tratto un film, anche un particolare dall'opera cinematografica: segno che la cultura attuale porta verso un'immagine di tipo mediatico.

I nostri esempi di autori, editori e copertine terminano qui: è una campionatura che ci auguriamo stimoli a continuare questi studi, i quali ci permettono di capire quanto sia importante per un testo l'immagine che lo accompagna e lo presenta. 


\section{Riferimenti bibliografici}

BARENGHI, Mario. Italo Calvino, le linee e i margini, 2007. Apud Cadioli Alberto, op. cit.

BATTISTINI, Andrea. "Le livree dei libri novecenteschi", in Poetiche. Rivista di letteratura, 2004. Apud Cadioli Alberto, op. cit.

BOLLATI, Giulio. http://www.einaudi.it/speciali/Einaudiani-Giulio-Bollati-su-Italo-Calvino

CADIOLI, Alberto. Le diverse pagine. Milano: Il Saggiatore, 2012.

CAVALLO, Guglielmo. CHARTIER, Roger (a cura di), Storia della lettura. Bari: Editori Laterza, 2009.

FERRERO, Ernesto. I migliori anni della nostra vita. Milano: Feltrinelli, 2005

FERRERO, Ernesto. http://www.einaudi.it/speciali/Einaudiani.-Ernesto-Ferrero-su-Giulio-Bollati

FILONI, Marco. "Scopertine”, in Venerdi di Repubblica, 26/02/2016

GENETTE, Gerard. Paratextos editoriais. Trad. Álvaro Faleiros. São Paulo: Ateliê, 2009.

GINZBURG, Natalia. Le piccole virtù. Torino: Einaudi, 1962

GRIMM, J. e W. Fiabe. Torino: Einaudi, 1970

PAVESE, Cesare. Antologia Einaudi 1948. Apud Cadioli Alberto, op. cit.

VITTORINI, Elio. Gli anni del "Politecnico". Lettere 1945-1951. Torino: Einaudi, 1977

Cinquant'anni di un editore. Le edizioni Einaudi negli anni 1933-1983. Torino: Einaudi 1983 http://www.internetculturale.it/opencms/opencms/it/pagine/mostre/pagina 190.html

Recebido em 14/04/2017

Aprovado em15/07/2017 